\title{
Editorial
}

\section{Special Issue based on the INFORMS Conference, Autumn 2003, Atlanta - Part 2}

This is Part 2 of a two-part issue dedicated to some of the papers presented at the INFORMS Conference in Atlanta, Autumn 2003, as part of the cluster of sessions sponsored by the Revenue Management and Pricing INFORMS Section. These papers represent a selection of new ideas and innovation which are emerging from academia and industry, and we hope you enjoy them.

\section{INFORMS PAPERS}

The issue starts with Natarajan Gautam of Pennsylvania State University, who considers a web-hosting application, where a web server hosts a website for a client organisation. Using a process of simulation, he shows that the dynamics between the three players (users, clients and web servers) demonstrates that the price and web server settings could either stabilise or oscillate between several points, indicating chaos. The contribution of the paper sets the ground work for further research and application, namely the opportunity for admission pricing, web caching mechanisms and dynamically executing control. Parijat Dube from IBM and colleagues consider the proposition of yield management and IT on demand through a means of determining an optimal reservation of resources in order to maximise expected revenue. The authors clearly demonstrate an application of yield management in a mainstream business field. Beat Burger and Matthias Fuchs consider the economic effects of a dynamic pricing strategy for the airline industry. They conclude that a dynamic pricing strategy not only influences revenue but also affects the cost structure of an airline and, hence, leads directly to a decrease in process complexity and transaction cost. Therefore, dynamic pricing seems to be the starting point for an efficient pull strategy, making dynamic pricing the new business model for the airline industry.

\section{RESEARCH AND PRACTICE PAPERS}

Opher Baron of Toronto University and colleagues propose a framework for analysing admission controls as pricing schemes for shared computer services. They use token bucket admission-control mechanisms as a pricing scheme. Concluding that the conceptual simplicity of such pricing schemes leads to the smoothing of the demand faced by the seller and this provides the seller with a mechanism to induce buyers to smooth demand. Sanket Jain of Virginia Polytechnic Institute and State University and $H$. Brian Bowman of IDeaS present an accurate method for measuring the gain attributable to the implementation of a revenue management system (RMS) for a hotel. The RMS makes use of three strategies to maximise revenue: length of stay, overbooking and rate controls. The method presented here is a direct measure of the benefits derived from the length-of-stay control due to the RMS. The measurement approach isolates and removes the impact of marketing programmes and economic factors from the
Journal of Revenue and Pricing Management, Vol. 4, No. 1, 2005, pp. 5-6

(C) Henry Stewart Publications, 1477-657X 
impact of the yield management solution to obtain the gain. The method presented provides a systematic and robust way of measuring the gain due to the length-ofstay controls. The contribution of the paper lies in the practical manner in which such a method can be used. Ben Vinod of Sabre presents a topical paper on revenue management and airline alliances, stating that revenue management poses unique challenges for members of an airline alliance that wants to synchronise decision making to maximise revenues across the alliance network. Ben's paper discusses the key challenges in alliance revenue management and its impacts on pricing and capacity planning. This is a cleverly written paper which challenges us to look at what the future may hold. Sheryl Kimes of Cornell University discusses how to make restaurant revenue management work, based upon adapting present strategies and introducing new ones.

This issue reflects the growing diversity of disciplines found in revenue management and pricing from operations research to restaurant management. This is a discipline which is still in its infancy and finding new applications. Enjoy the papers.

Georgia Perakis Americas Editor

Ian Yeoman Editor 